\title{
Deterministic Computation of Pseudorandomness in Sequences of Cryptographic Application*
}

\author{
A. Fúster-Sabater ${ }^{1}$, P. Caballero-Gil ${ }^{2}$, and O. Delgado-Mohatar ${ }^{1}$ \\ 1 Institute of Applied Physics, C.S.I.C., Serrano 144, 28006 Madrid, Spain \\ amparo@iec.csic.es, oscar.delgado@iec.csic.es \\ 2 Fac. of Maths, D.E.I.O.C., University of La Laguna, 38271 Tenerife, Spain \\ pcaballe@ull.es
}

\begin{abstract}
An easy method of checking balancedness degree as well as run quantification in sequences obtained from LFSR-based keystream generators has been developed. The procedure is a deterministic alternative to the traditional application of statistical tests. The computation method allows one to check deviation of balancedness and run distribution goodness from standard values. The method here developed can be considered as a first selective criterium for acceptance/rejection of this type of generators of cryptographic application.
\end{abstract}

Keywords: Pseudorandomness, bit-string, balancedness, run dirtribution, cryptography.

\section{Introduction}

Transmission of sensitive information between two interested parties needs several security requirements (confidentiality, integrity, non repudiation, authentication ...) that can be satisfied by means of design, assessment and implementation of cryptographic algorithms and security protocols.

Confidentiality makes use of an encryption function currently called cipher that converts the plaintext into the ciphertext. Ciphers are usually divided into two large classes: stream ciphers and block-ciphers. Stream ciphers are very fast (in fact, the fastest among the encryption procedures) so they are implemented in many technological applications e.g. algorithms A5 in GSM communications 7] or the encryption system E0 used in the Bluetooth specifications [1. Stream ciphers try to imitate the ultimate one-time pad cipher and are supposed to be good pseudorandom generators capable of stretching a short secret seed (the secret key) into a long sequence of seemingly random bits (the keystream sequence). This sequence is then XORed with the plaintext in order to obtain the

\footnotetext{
* Work supported by CDTI (Spain) and the companies INDRA, Unión Fenosa, Tecnobit, Visual-Tools, BrainStorm, SAC and TechnoSafe under programme CenitHESPERIA as well as supported by Ministry of Science and Innovation and European FEDER Fund under Project TIN2008-02236/TSI.
}

G. Allen et al. (Eds.): ICCS 2009, Part I, LNCS 5544, pp. 621-630, 2009.

(C) Springer-Verlag Berlin Heidelberg 2009 
ciphertext. Most generators producing keystream sequence are based on Linear Feedback Shift Registers (LFSRs) e.g. see [6] and [8]. The pseudorandom output sequence is a periodic sequence generated as the image of a nonlinear Boolean function in the LFSR stages.

Desirable properties for such sequences can be enumerated as follows: 1) large period, 2) large linear complexity, 3) good statistical properties. There are wellknown proposals [2], 44, 9], [1], 12] for which conditions 1) and 2) above are perfectly satisfied. Nevertheless, how to generate sequences with good statistics is a feature that even now remains quite diffuse, see 3 .

Balancedness and adequate distribution of $1^{\prime} s$ and $0^{\prime} s$ in the output sequence are necessary (although not sufficient) conditions that every keystream generator must satisfy. Roughly speaking, a binary sequence is balanced if it has approximately the same number of $1^{\prime} s$ as $0^{\prime} s$. On the other hand, a run of $1^{\prime} s\left(0^{\prime} s\right)$ of length $k$ is defined as a succession of $k$ consecutive $1^{\prime} s\left(0^{\prime} s\right)$ between two $0^{\prime} s$ $\left(1^{\prime} s\right)$. The runs of $1^{\prime} s$ are called blocks while the runs of $0^{\prime} s$ are called gaps. It is a well known fact [6] that in a pseudorandom binary sequence of period $T$ there are $T / 2$ runs distributed as follows: half the runs have length 1 , one quarter of the runs length 2, one eighth of the runs length 3, and so forth. Moreover, half the runs of any length are gaps, the other half are blocks. That is to say, in a pseudorandom binary sequence the number and distribution of digits is perfectly quantified.

Due to the long period of the output sequence $\left(\simeq 10^{77}\right)$ in cryptographic applications, it is unfeasible to produce an entire cycle of such a sequence and then analyze the number and distribution of $1^{\prime} s$ and $0^{\prime} s$. Therefore, in practice, portions of the output sequence are chosen randomly and different statistical tests [11] (monobit test, run test, poker test, serial test ... ) are applied to all these subsequences. Nevertheless, passing the previous tests merely provides probabilistic evidence that the LFSR-based keystream generator produces a sequence with certain characteristics of pseudorandomness.

In this work, deterministic expressions to compute the degree of balancedness and number of runs in one period of the output sequence are proposed. If the computed values are not in the expected range, then the generator must be rejected.

\section{Notation and Basic Concepts}

Any $L$-variable Boolean function can be expressed canonically in terms of its minterms [5], that is the logic product of the $L$ variables $\left(a_{1}, a_{2}, \ldots, a_{L}\right)$ where each variable can be in its true or complementary form. Examples of minterms of $L$ variables are:

$$
a_{1} a_{2} \ldots a_{L}, \quad \bar{a}_{1} a_{2} \ldots \bar{a}_{L}, \quad \bar{a}_{1} \bar{a}_{2} \ldots \bar{a}_{L}
$$

where the superposition of variables represents the logic product. In addition, any $L$-variable Boolean function can be uniquely expressed in Algebraic Normal Form (A.N.F.) or Muller expansion [10] by means of the sum exclusive-OR of 
logic products of different orders in the $L$ variables. A simple example of Boolean function in A.N.F. is:

$$
f\left(a_{1}, a_{2}, \ldots, a_{L}\right)=a_{1} a_{2} \oplus a_{2} a_{L-1} \oplus a_{L}
$$

where $\oplus$ represents the exclusive-OR logic operation.

In mathematical terms, a LFSR-based generator is a $L$-variable nonlinear Boolean function, $F: G F(2)^{L}-\{0\} \rightarrow G F(2)$, whose $L$ input-variables are the stages of the LFSRs. At each clock pulse the LFSRs generate new stage contents that will be the new input-variables of $F$. In this way, the generator produces the successive bits of the output sequence or generated sequence. A LFSR-based keystream generator is nothing but a nonlinear Boolean function $F$ given in its A.N.F. Moreover, the LFSRs involved in this kind of generator are maximal length-LFSRs [6], that is LFSRs whose characteristic polynomials are primitive. In this case, their output sequences are called $P N$-sequences. Balancedness and run distribution of $P N$-sequences have been extensively studied in the literature. See for example [6] and [13].

Let $A$ be an arbitrary maximal length-LFSR of length $L_{A}$ and $a_{i}\left(i=1, \ldots, L_{A}\right)$ the binary content of the $i$-th LFSR stage. A minterm of $L_{A}$ variables is denoted by $A_{i \ldots j}$ whether such a minterm includes the variables $a_{i} \ldots a_{j}$ in their true form while the other variables are in complementary form.

Let $\Lambda_{L}$ denote the set of $L$-variable Boolean functions in A.N.F. and $\Phi_{F}$ the minterm function of $F$. In fact, $\Phi_{F}: \Lambda_{L} \rightarrow \Lambda_{L}$, such that, given $F, \Phi_{F}$ substitutes every term of $F$ by its corresponding minterm. For a nonlinear function in A.N.F., e.g. $F\left(a_{1}, a_{2}, a_{3}\right)=a_{1} a_{2} a_{3} \oplus a_{1} a_{3} \oplus a_{2} \oplus a_{3}$, we have:

$$
\Phi_{F}=A_{123} \oplus A_{13} \oplus A_{2} \oplus A_{3} .
$$

On the other hand, every minterm considered as a generator applied to the $L_{A}$ stages of $A$ generates a canonical sequence with an unique 1 and period $T=2^{L_{A}}-1$, see 11 .

Once the nonlinear function $F$ given in its A.N.F. has been converted into its minterm expansion, the basic ideas of this work can be summarized as follows:

1. The number of minterms in the representation of $F$ equals the number of $1^{\prime} s$ in the output sequence as every minterm provides the generated sequence with an unique 1 .

2. The contiguity of such minterms in the ordered minterm succession determines the run distribution in the output sequence.

Let us now generalize the previous statements to more than one LFSR. Let $A, B, \ldots, Z$ be $N$ LFSRs whose lengths are respectively $L_{A}, L_{B}, \ldots, L_{Z}$ (supposed $\left.G C D\left(L_{i}, L_{j}\right)=1, \quad i \neq j\right)$. We denote by $a_{i}\left(i=1, \ldots, L_{A}\right), b_{j}(j=$ $\left.1, \ldots, L_{B}\right), \ldots, z_{k}\left(k=1, \ldots, L_{Z}\right)$ their corresponding stages. The global minterms associated with the generator have now $L_{A}+L_{B}+\ldots+L_{Z}$ variables and are of the form, e.g. $A_{i j} B_{p q r} \ldots Z_{s}$, that is to say the ordered product of the individual minterms of each LFSR. As before every global minterm considered as 
a generator applied to the stages of the LFSRs generates a sequence with an unique 1 and period $T=\left(2^{L_{A}}-1\right)\left(2^{L_{B}}-1\right) \ldots\left(2^{L_{Z}}-1\right)$ [11. In brief, every LFSR-based generator can be expressed in terms of its global minterms as well as global minterms determine balancedness and run distribution in the output sequence.

\section{From A.N.F. to Global Minterm Expansion}

Previously to the conversion algorithm, the following facts are introduced:

Fact 1: For every Boolean function $F$ the following equality holds [10]

$$
F=\Phi_{F} \circ \Phi_{F}
$$

where the symbol $\circ$ denotes the composition of functions.

Fact 2: For every LFSR $A$, the exclusive-OR of all the minterms [11] is:

$$
A_{12 \ldots L_{A}} \oplus A_{12 \ldots L_{A}-1} \oplus \ldots \oplus A_{2 \ldots L_{A}} \oplus \ldots \oplus A_{L_{A}} \oplus \ldots \oplus A_{2} \oplus A_{1}=1 .
$$

The previous equation can be rewritten as:

$$
A_{1}^{\prime} \oplus A_{1}=1 .
$$

On the other hand, the total number of terms in (2) is:

$$
\sum_{i=1}^{L_{A}}\left(\begin{array}{c}
L_{A} \\
i
\end{array}\right)=2^{L_{A}}-1
$$

while the number of terms in $A_{1}=a_{1} \bar{a}_{2} \ldots \bar{a}_{L_{A}}$ is [10]:

$$
N_{t}\left(A_{1}\right)=2^{L_{A}-1} \text {. }
$$

Thus, the number of terms in $A_{1}^{\prime}$ will be:

$$
N_{t}\left(A_{1}^{\prime}\right)=2^{L_{A}-1}-1
$$

Appropriate notation will be used for the rest of LFSRs.

\subsection{Conversion Algorithm}

Input: $N$ (number of LFSRs), $L_{A}, L_{B}, \ldots, L_{Z}$ (lengths of the LFSRs) and a nonlinear function $F$ in A.N.F.

For instance, $N_{Z}=2, L_{A}=2, L_{B}=3$ and $F\left(a_{1}, a_{2}, b_{1}, b_{2}, b_{3}\right)=a_{1} b_{1}$.

- Step 1: Compute $\Phi_{F}$

$$
\Phi_{F}=A_{1} B_{1}
$$


- Step 2: Substitute every minterm by its corresponding function in A.N.F. and cancel common terms (if there exist)

$$
\begin{gathered}
\Phi_{F}=\left(a_{1} a_{2} \oplus a_{1}\right)\left(b_{1} b_{2} b_{3} \oplus b_{1} b_{2} \oplus b_{1} b_{3} \oplus b_{1}\right)= \\
a_{1} a_{2} b_{1} b_{2} b_{3} \oplus a_{1} a_{2} b_{1} b_{2} \oplus a_{1} a_{2} b_{1} b_{3} \oplus a_{1} a_{2} b_{1} \oplus \\
a_{1} b_{1} b_{2} b_{3} \oplus a_{1} b_{1} b_{2} \oplus a_{1} b_{1} b_{3} \oplus a_{1} b_{1} .
\end{gathered}
$$

- Step 3: Compute $F\left(a_{i}, b_{j}\right)=\Phi_{F} \circ \Phi_{F}$

$$
\begin{gathered}
F\left(a_{i}, b_{j}\right)=\Phi_{F} \circ \Phi_{F}=A_{12} B_{123} \oplus A_{12} B_{12} \oplus A_{12} B_{13} \oplus A_{12} B_{1} \oplus \\
A_{1} B_{123} \oplus A_{1} B_{12} \oplus A_{1} B_{13} \oplus A_{1} B_{1} .
\end{gathered}
$$

Output: $F$ expressed in terms of its global minterms.

Once the function $F$ has been expressed in terms of its minterms, balancedness and run distribution in the output sequence can be analyzed.

\section{Balancedness in the Keystream Sequence}

The number of 1's in the generated sequence coincides with the number of global minterms in the expression of $F$ or, equivalently, the number of terms in $\Phi_{F}$ (Step 2). An illustrative example of application is presented.

\subsection{A Numerical Example}

Example 1: Let $A, B, C$ be three LFSRs of lengths $L_{A}, L_{B}, L_{C}$ respectively. The generating function is chosen:

$$
F=a_{1} b_{1} \oplus b_{1} c_{1} \oplus c_{1}
$$

(Geffe's generator [13]) and the minterm function $\Phi_{F}$ is computed:

$$
\begin{aligned}
\Phi_{F}= & A_{1} B_{1} \oplus B_{1} C_{1} \oplus C_{1} \\
= & A_{1} B_{1}\left(C_{1}^{\prime} \oplus C_{1}\right) \oplus\left(A_{1}^{\prime} \oplus A_{1}\right) B_{1} C_{1} \oplus \\
& \oplus\left(A_{1}^{\prime} \oplus A_{1}\right)\left(B_{1}^{\prime} \oplus B_{1}\right) C_{1} \\
= & A_{1} B_{1}\left(C_{1}^{\prime} \oplus C_{1}\right) \oplus\left(A_{1}^{\prime} \oplus A_{1}\right) B_{1}^{\prime} C_{1} .
\end{aligned}
$$

The number of $1^{\prime} s$ in the output sequence can be directly obtained by counting the number of terms in $\Phi_{F}$ via the equations (5) and (6). According to this simple rule, the number of $1^{\prime} s$ in the output sequence obtained from a Geffe's generator is given by:

$$
\text { No. } 1^{\prime} s=2^{L_{A}-1} 2^{L_{B}-1}\left(2^{L_{C}}-1\right)+\left(2^{L_{A}}-1\right)\left(2^{L_{B}-1}-1\right) 2^{L_{C}-1} .
$$

Remark that the previous expression is function exclusively of the lengths of the LFSRs. In a practical range, we say $L_{i} \simeq 120$, and keeping in mind that the sequence period is $T=\left(2^{L_{A}}-1\right)\left(2^{L_{B}}-1\right)\left(2^{L_{C}}-1\right)$, the number of $1^{\prime} s$ in the output sequence is:

$$
\text { No. } 1^{\prime} s \simeq T / 2 .
$$

Consequently, the generated sequence is balanced. 


\section{Run Distribution in the Keystream Sequence}

The computation of runs in the output sequence is based on the following result.

Proposition 1. Let us consider the ordered minterm succession of a maximal length-LFSR of length $L$. If the minterms including an arbitrary index $i$ are replaced by 1 and the minterms not including the index $i$ are replaced by 0 , then the resulting binary sequence is the reverse version of the $P N$-sequence generated by the LFSR.

The previous result is a straight application of the LFSR linear recurrence relationship given by its characteristic polynomial. Thus, a minterm succession can be treated as a $P N$-sequence. Now, keep in mind the following remarks:

1. The number of runs of any length of a $P N$-sequence is perfectly quantified. Indeed, each $m$-gram (every one of the $2^{m}$ possible configurations of $m$ bits $(m=1, \ldots, L))$ will appear exactly $2^{L-m}$ times throughout the $P N$-sequence except for the $L$-gram $00 \ldots 0$ that will not appear any time.

2. In the global minterm succession each $m$-gram of any LFSR will coincide once with each one of the $m$-grams of the other LFSRs.

Based on these considerations, the computation of runs in the output sequence can be carried out as it is shown in the following example.

Example 2: For two LFSRs, $A$ and $B$, of lengths $L_{A}$ and $L_{B}$ respectively $\left(L_{A}\right.$ $\left.<L_{B}\right)$ and generating function $F=a_{1} b_{1}$, we proceed:

$$
\begin{gathered}
\Phi_{F}=A_{1} B_{1}=\left(a_{1} \oplus a_{1} a_{2} \oplus \ldots \oplus a_{1} a_{2} \ldots a_{L_{A}}\right)\left(b_{1} \oplus b_{1} b_{2} \oplus \ldots \oplus b_{1} b_{2} \ldots b_{L_{B}}\right) \\
F=\Phi_{F} \circ \Phi_{F}=\left(A_{1} \oplus A_{12} \oplus \ldots \oplus A_{12 \ldots L_{A}}\right)\left(B_{1} \oplus B_{12} \oplus \ldots \oplus B_{12 \ldots L_{B}}\right)
\end{gathered}
$$

Notice that the minterm expansion of $F$ will only include products of individual minterms with the index 1 . Let us now introduce the following notation:

$Y$ denotes an arbitrary minterm of $A$ or $B$ including the index 1 .

$N$ denotes an arbitrary minterm of $A$ or $B$ not including the index 1 .

$\operatorname{Sec} A$ denotes the ordered succession of minterms of $A$ in format $Y / N$.

$\operatorname{Sec} B$ denotes the ordered succession of minterms of $B$ in format $Y / N$.

It is clear that an 1 in the output sequence corresponds to a minterm product $Y Y$ (for example, $A_{1} B_{12}$ ) while a 0 in the output sequence corresponds to the minterm products $Y N, N Y$ or $N N$ (for example, $A_{1} B_{2}, A_{23} B_{13}$ or $A_{3} B_{2}$ ). See the formation rule in Table 1 .

Now we can compute the number of runs of different lengths.

Table 1. Global minterm formation rule for $F$ in Example 1

\begin{tabular}{|c|c|c|}
\hline SecB & SecA & Output bit \\
\hline $\mathrm{Y}$ & $\mathrm{Y}$ & 1 \\
$\mathrm{~N}$ & $\mathrm{Y}$ & 0 \\
$\mathrm{Y}$ & $\mathrm{N}$ & 0 \\
$\mathrm{~N}$ & $\mathrm{~N}$ & 0 \\
\hline
\end{tabular}




\subsection{Runs of Length 1}

Blocks: They are runs of the form "0 10 " that come from minterm structures

$$
\begin{aligned}
& S e c B: * Y * \\
& \text { SecA }: * Y *
\end{aligned}
$$

The symbol $*$ denotes $Y$ or $N$. The 3 -gram $N Y N$ will appear $2^{L_{A}-3}$ times in $\operatorname{Sec} A$ and $2^{L_{B}-3}$ times in $S e c B$, the 2 -gram $N Y *$ will appear $2^{L_{A}-2}$ times in $\operatorname{Sec} A$ and $2^{L_{B}-2}$ times in $\operatorname{Sec} B$, and so forth.

The different configurations of minterms able to generate a block of length 1 are depicted in Table 2 at columns with heading "Configurations". The columns with heading "No. of config." show the number of times that such configurations appear on their corresponding minterm sequences.

Thus, the number of blocks of length 1 will be the sum of all suitable configurations multiplied by the number of times that such configurations appear

$$
N_{B}(1)=\left(2^{L_{B}-1}+2 \cdot 2^{L_{B}-2}+2^{L_{B}-3}\right) 2^{L_{A}-3} .
$$

Gaps: They are runs of the form "1 01 " that come from minterm structures

$$
\begin{aligned}
& \text { SecB }: Y * Y \\
& \text { SecA }: Y * Y
\end{aligned}
$$

The different configurations of minterms able to generate a gap of length 1 are depicted in Table 3 .

Thus, the number of gaps of length 1 will be the sum of all suitable configurations multiplied by the number of times that such configurations appear

$$
N_{G}(1)=\left(2^{L_{B}-3}+2^{L_{B}-2}\right) 2^{L_{A}-3} .
$$

Table 2. Configurations of minterms producing blocks of length 1

\begin{tabular}{|c|c|c|c|c|}
\hline & Configuration & No. of config. & Configuration & No. of config. \\
\hline SecB & $* Y *$ & $2^{L_{B}-1}$ & $* Y N$ & $2^{L_{B}-2}$ \\
SecA & $N Y N$ & $2^{L_{A}-3}$ & $N Y Y$ & $2^{L_{A}-3}$ \\
\hline \hline SecB & $N Y *$ & $2^{L_{B}-2}$ & $N Y N$ & $2^{L_{B}-3}$ \\
SecA & $Y Y N$ & $2^{L_{A}-3}$ & $Y Y Y$ & $2^{L_{A}-3}$ \\
\hline
\end{tabular}

Table 3. Configurations of minterms producing gaps of length 1

\begin{tabular}{|l|c|c|c|c|}
\hline & Configuration & No. of config. & Configuration & No. of config. \\
\hline SecB & $Y N Y$ & $2^{L_{B}-3}$ & $Y * Y$ & $2^{L_{B}-2}$ \\
SecA & $Y Y Y$ & $2^{L_{A}-3}$ & $Y N Y$ & $2^{L_{A}-3}$ \\
\hline
\end{tabular}




\subsection{Runs of Length $n$}

The procedure can be generalized in order to compute the number of runs of length $n\left(n=1, \ldots, L_{A}-2\right)$.

Blocks: They are runs of the form " $01 \ldots 10$ " (with $n$ consecutive $1^{\prime} s$ ) coming out from minterm structures

$$
\begin{aligned}
& \operatorname{Sec} B: * Y \ldots Y * \\
& \operatorname{Sec} A: * Y \ldots Y *
\end{aligned}
$$

with $n$ minterms $Y$ in both sequences. The different configurations able to generate a block of length $n$ and their number are depicted in Table 4 .

Thus, the number of blocks of length $n$ will be:

$$
N_{B}(n)=\left(2^{L_{B}-(n+2)}+2 \cdot 2^{L_{B}-(n+1)}+2^{L_{B}-n}\right) 2^{L_{A}-(n+2)} .
$$

Gaps: They are runs of the form "1 $0 \ldots 01 "$ (with $n$ consecutive $0^{\prime} s$ ) coming out from minterm structures

$$
\begin{aligned}
& \operatorname{Sec} B: Y * \ldots * Y \\
& \operatorname{Sec} A: Y * \ldots * Y
\end{aligned}
$$

with $n$ symbols $*$ in both sequences. Notice that in $S e c A$ there will be $2^{n}$ different configurations able to generate a gap of length $n$ ranging from $Y N \ldots N Y$ up to $Y Y \ldots Y Y$. Some of such configurations and their number are depicted in Table 5 .

Thus, the number of gaps of length $n$ will be:

$$
N_{G}(n)=\left(\sum_{i=0}^{n}\left(\begin{array}{c}
n \\
i
\end{array}\right) 2^{L_{B}-(n+2-i)}\right) \cdot 2^{L_{A}-(n+2)} .
$$

Therefore, the number of runs of any length up to $L_{A}-2$ can be easily computed in the proposed example. Equations (11) and (12) give us the exact

Table 4. Configurations of minterms producing blocks of length $\mathrm{n}$

\begin{tabular}{|c|c|c|c|c|}
\hline & Configuration & No. of config. & Configuration & No. of config. \\
\hline SecB & $N Y \ldots Y N$ & $2^{L_{B}-(n+2)}$ & $* Y \ldots Y N$ & $2^{L_{B}-(n+1)}$ \\
SecA & $Y Y \ldots Y Y$ & $2^{L_{A}-(n+2)}$ & $N Y \ldots Y Y$ & $2^{L_{A}-(n+2)}$ \\
\hline \hline SecB & $N Y \ldots Y *$ & $2^{L_{B}-(n+1)}$ & $* Y \ldots Y *$ & $2^{L_{B}-n}$ \\
SecA & $Y Y \ldots Y N$ & $2^{L_{A}-(n+2)}$ & $N Y \ldots Y N$ & $2^{L_{A}-(n+2)}$ \\
\hline
\end{tabular}

Table 5. Configurations of minterms producing gaps of length $\mathrm{n}$

\begin{tabular}{|c|c|c|c|c|}
\hline & Configuration & No. of config. & Configuration & No. of config. \\
\hline SecB & $Y * \ldots * Y$ & $2^{L_{B}-2}$ & $Y N \ldots N Y$ & $2^{L_{B}-(n+2)}$ \\
SecA & $Y N \ldots N Y$ & $2^{L_{A}-(n+2)}$ & $Y Y \ldots Y Y$ & $2^{L_{A}-(n+2)}$ \\
\hline
\end{tabular}


Table 6. Numerical example

\begin{tabular}{|c|c|c|c|c|}
\hline $\mathrm{n}$ & No. of blocks & \%Deviation(blocks) & No. of gaps & \%Deviation(gaps) \\
\hline 1 & 4608 & $13,8 \%$ & 1536 & $62,0 \%$ \\
2 & 1152 & $43,1 \%$ & 1152 & $43,1 \%$ \\
3 & 288 & $71,5 \%$ & 864 & $14,6 \%$ \\
4 & 72 & $85,7 \%$ & 648 & $28,1 \%$ \\
5 & 18 & $92,8 \%$ & 486 & $92,1 \%$ \\
\hline
\end{tabular}

number of blocks and gaps that can be found in the output sequence. Remark that $N_{B}$ and $N_{G}$ depend exclusively on the LFSR's lengths $L_{A}$ and $L_{B}$ and on the run length $(n)$. There is no dependency on the primitive characteristic polynomials. Consequently, different LFSRs of the same length primitive characteristic polynomials will produce output sequences with the same number of blocks and gaps.

According to these expressions, it can be seen that the analyzed function $F$ does not match the expected values. For a numerical example $L_{A}=7$ and $L_{B}=8$, see results in Table 6. For $n=1, N_{B}>N_{G}$. For $n=2$, equations (11) and (12) coincide. For $n \geq 3, N_{B}<N_{G}$. As expected, there are more gaps than blocks because the formation rule in Table 1 is not balanced.

The upper limit $L_{A}-2, L_{A}$ being the length of the shortest LFSR, follows from the fact that blocks and gaps of length $n$ include $n+2$ bits but we can only guarantee the presence of at most $L_{A}$-grams. At any rate, the designer of keystream generators is basically interested in the runs of low length (e.g. up to length 15) while in, for instance, cryptographic applications every LFSR length takes values in the range $L_{i} \simeq 120$.

\section{Conclusions}

An easy and efficient method of checking the degree of balancedness and number of runs in the output sequence of keystream generators has been presented. From the concept of global minterm, it is possible to derive general expressions for the number of $1^{\prime} s\left(0^{\prime} s\right)$ as well as for the number of runs in the output sequence of any LFSR-based generator. Then, the obtained values are compared with the expected values for a sequence to be pseudorandom. The result of this comparison implies the assessment of such a sequence generator.

In this work, such a method has been applied exclusively to nonlinear generating functions. Nevertheless, these ideas concerning the analysis of the global minterms seem to be suitable for more general pattern generators. Consider, for instance, the multiple-speed generators [1] that can be expressed in terms of a more complex generating function or the shrinking generator [1] whose global minterms can be obtained by removing certain individual minterms from one of the LFSRs. In both cases, the developed method can be adapted and applied to these schemes in order to evaluate certain aspects of pseudorandomness in the generated sequences. 


\section{References}

1. Bluetooth, Specifications of the Bluetooth system, Version 1.1 (February 2001), http://www.bluetooth.com/

2. Caballero-Gil, P., Fúster-Sabater, A.: A Wide Family of Nonlinear Filter Functions with a Large Linear Span. Inf. Sci. 164, 197-207 (2004)

3. Fúster-Sabater, A., García-Mochales, P.: On the Balancedness of nonlinear generators of binary sequences. Inf. Process. Lett. 85, 111-116 (2003)

4. Fúster-Sabater, A., Caballero-Gil, P.: Linear solutions for cryptographic nonlinear sequence generators. Phys. Lett. A. 369, 432-437 (2007)

5. Grassman, W.K., Trembley, J.P.: Logic and Discrete Mathematics. Prentice-Hall, New York (1996)

6. Golomb, S.W.: Shift Register-Sequences. Aegean Park Press, Laguna Hills (1982)

7. GSM, Global Systems for Mobile Communications, http://cryptome.org/gsm-a512.htm

8. Kanso, A.: Clock-Controlled Shrinking Generator of Feedback Shift Registers. In: Safavi-Naini, R., Seberry, J. (eds.) ACISP 2003. LNCS, vol. 2727, pp. 443-451. Springer, Heidelberg (2003)

9. Klapper, A.: Large Families of Sequences with Near Optimal Correlations and Large Linear Span. IEEE Trans. Inf. Theory. 42, 1241-1248 (1996)

10. Mange, D.: Analysis and Synthesis of Logic Systems. Artech House, Inc., Norwood (1986)

11. Menezes, A.J., et al.: Handbook of Applied Cryptography. CRC Press, New York (1997)

12. Shparlinski, I.E.: On the Linear Complexity of the Power Generator. Design, Codes and Cryptography 23, 5-10 (2001)

13. Simmons, G.J. (ed.): Contemporary Cryptology: The Science of Information Integrity. IEEE Press, New York (1991) 